\title{
AJUSTAMENTO PSICOLÓGICO E PERSPECTIVA DE VELHICE PESSOAL EM ADULTOS COM DEFICIÊNCIA FÍSICA
}

\author{
Marineia Crosara de Resende* \\ Anita Liberalesso Neri ${ }^{\#}$
}

\begin{abstract}
RESUMO. Foram investigadas relações entre senso de ajustamento psicológico e perspectiva de velhice em adultos e idosos com deficiência física. Participaram 90 pessoas, de ambos os sexos, com idade entre 25 e 84 anos, que responderam aos instrumentos: questionários sociodemografico e sobre a deficiência física; Inventário Sheppard de Atitudes em Relação à Velhice Pessoal; Escala de Desenvolvimento Pessoal (ajustamento psicológico). Os índices de ajustamento pessoal foram de moderados a altos, mas as mulheres pontuaram mais baixo. Para toda a amostra, quanto maior o senso de ajustamento, mais positiva a perspectiva de velhice pessoal. Os com deficiência congênita e os mais ajustados psicologicamente mostraram perspectivas mais positivas de velhice pessoal. Concluiu-se que envelhecer com uma deficiência física é um processo que exige competência adaptativa e resiliência aos eventos de vida e aos desafios acarretados pela deficiência.
\end{abstract}

Palavras-chave: Envelhecimento; deficiência física; ajustamento psicológico.

\section{PSYCHOLOGICAL ADJUSTMENT AND PERSONAL AGING PERSPECTIVE IN ADULTS AND OLDER ADULTS WITH PHYSICAL DISABILITY}

\begin{abstract}
We carried out an investigation to analyze the relationships between perceived psychological adjustment and perspective of personal aging in adult and aged people with physical disability. Materials and Method: 90 participants, both genders, aged 25 to 84 , answered questionnaires asking for information on socio demographic characteristics and disability; the Sheppard Inventory of attitudes toward own aging; and a scale assessing perceived psychological adjustment. Results: The indexes of personal adjustment were moderate and high. The women had the lowest scores. There were observed positive correlations between positive psychological adjustment and positive attitudes toward own aging. Those that had congenital disability and reported higher psychological adjustment showed more positive perspectives of personal aging. Conclusion: Aging with physical disability is a complex process that demands adaptive competence, and resilience to cope with life events and the personal, social and environmental challenges produced by disability.
\end{abstract}

Key words: Aging; physical disability; psychological adjustment.

\section{AJUSTE PSICOLÓGICO E LA PERSPECTIVA PERCIBIDA DEL ENVEJECIMIENTO PERSONAL EN ADULTO Y ADULTOS MOYORES CON DISCAPACIDAD FÍSICA}

RESUMEN. Realizamos una investigación para analizar las relaciones entre el ajuste psicológico y la perspectiva percibida del envejecimiento personal en adulto y adultos mayores con discapacidad física. Materiales y método: 90 participantes, ambos géneros, adultos con 25 a 84 respondieron a cuestionarios contestados pidiendo la información sobre características socio demográficas y discapacidad; el inventario de Sheppard de actitudes acerca del propio envejecimiento; y una escala que determina el ajuste psicológico percibido. Resultados: Los índices del ajuste personal eran moderados y altos. Las mujeres tenían las cuentas más bajas. Había correlaciones positivas observadas entre el ajuste psicológico positivo y las actitudes positivas acerca del propio envejecimiento. Los que tenían discapacidad congénita y el ajuste psicológico más alto divulgado demostraron perspectivas más positivas del envejecimiento personal. Conclusión: El envejecer con discapacidad física es un proceso complejo que exige capacidad adaptante, y resistencia para hacer frente a acontecimientos de vida y a los desafíos personales, sociales y ambientales producidos por la discapacidad..

Palabras clave: Envejecimiento; ajuste psicológico; discapacidad física.

\footnotetext{
* Psicóloga, Professora Adjunto Nível 1 da Universidade Federal de Uberlândia.

\# Psicóloga, Livre-docente. Professora Titular vinculada ao Programa de Pós-graduação em Gerontologia, Faculdade de Educação da Universidade Estadual de Campinas-Unicamp.
} 
Envelhecer bem requer ajustamento pessoal e social, que pode ser comprometido por condições deficitárias de saúde e educação ao longo do curso de vida. Se o indivíduo tiver alguma deficiência física congênita ou adquirida antes da velhice, suas condições de desenvolver-se e envelhecer com sucesso sofrerão prejuízo maior ou menor e mais ou menos controlável, dependendo da extensão e da natureza da sua deficiência, dos recursos de apoio que o ambiente sociocultural lhe oferecer ao longo de toda a vida e de seus recursos psicológicos.

\section{DEFICIÊNCIA FÍSICA E AJUSTAMENTO}

A partir da síntese de teorias clássicas sobre a personalidade, Ryff (1989) propôs um modelo multidimensional de bem-estar psicológico, correspondente a funcionamento psicológico positivo como sinônimo de ajustamento, e uma condição do self relacionada a seis domínios do funcionamento psicológico: autoaceitação, relações positivas com os outros, autonomia, domínio sobre o ambiente, propósito na vida e crescimento pessoal.

A autoaceitação significa uma atitude positiva do indivíduo em relação a si próprio e a seu passado; significa reconhecer e aceitar suas características positivas e negativas. $\mathrm{O}$ sentimento de aceitação gera estima, confiança e segurança em si e nos outros (Ryff, 1989). A aceitação da deficiência é definida como um dos melhores indicadores de ajustamento positivo após uma deficiência e caracterizada por: a) capacidade do indivíduo com deficiência de perceber valor em habilidades e metas que não foram perdidas em consequência da deficiência; b) avaliação do próprio valor, de atributos e capacidades, baseada não apenas em aspectos físicos, mas também em outros (por exemplo, persistência, inteligência); c) mesmo quando o indivíduo é influenciado por percepções, atitudes e linguagem de outras pessoas, foca a sua própria atitude em relação à deficiência, enxergando-a como mais uma de suas características, e não a única; d) em seu nível mais básico, ocorre quando o indivíduo reconhece o valor único da junção de suas características e habilidades, mais do que quando faz comparações com padrões externos e frequentemente inatingíveis (Elliott, Kyrlo \& Rivera, 2002, citado por Schmitt \& Elliott, 2004).

Ter relações positivas diz respeito a manter com os outros uma relação de qualidade, uma relação calorosa, satisfatória e verdadeira; preocupar-se com o bem-estar do outro e ser capaz de manter relações afetuosas e agradáveis, sejam elas familiares, de intimidade ou de amizade (Ryff, 1989). Relações sociais significativas permitem o desenvolvimento do self, dão sentido às experiências e podem oferecer apoio, importantes elementos no processo de adaptação, principalmente em momentos de transição da vida adulta. No caso específico das pessoas com deficiência física, o apoio social pode tornar-se mais significativo. $\mathrm{O}$ indivíduo se sente mais amado e estimado e com sensação de controle sobre sua própria vida (Nogueira, 2001).

A autonomia implica em ser autodeterminado e independente, mesmo que a pessoa necessite de apoio para operacionalizar suas escolhas e, às vezes, até para o exercício de atividades de vida diária. Implica em tomar decisões de acordo com o que acredita ser melhor, dentro de seus próprios padrões, e em não ser influenciado por pressões sociais para pensar e agir (Ryff, 1989). O conceito de autonomia pode ser comparado aos conceitos de autodeterminação, independência, locus de controle interno, individuação e regulação interna do comportamento, aos quais se subordinam as crenças de que os pensamentos e ações são próprios à pessoa e não são determinados por causas independentes do próprio controle. Está relacionado ao conceito de liberdade, e, como teorizou Kant, é a capacidade de autonomia que traz aos seres humanos dignidade (Cristopher, 1999).

Segundo Ryff (1989), o senso de domínio sobre o ambiente significa ter competência para manejar o ambiente, usufruir as oportunidades que surgem ao redor, apresentar habilidades para escolher ou criar contextos adequados às suas necessidades e valores, eliminar barreiras e fazer adaptações pessoais ou no ambiente, quando necessárias. É preciso que seja compatível com as capacidades físicas e com as competências comportamentais das pessoas para permitir melhor adaptação. Pessoas que apresentam maior habilidade para manejar o ambiente têm senso de controle mais elevado. De acordo com Sirois, Davis e Morgan (2006), teorias de adaptação cognitiva à adversidade sugerem que crenças de controle têm um papel central na vida do indivíduo. Segundo Folkman (1984), o papel do controle no ajustamento vai depender do significado das crenças de controle em cada situação, e quando as oportunidades de controle são limitadas em determinado contexto, a pessoa irá buscar algum aspecto da própria situação onde ela tenha esse senso de controle, para se fortalecer. Questões sobre controle podem ser particularmente importantes no contexto de débitos na saúde, tendo-se em vista que em alguns casos de doenças crônicas as oportunidades de controle estão limitadas, mesmo que sejam sobre o ambiente (Sirois, Davis \& Morgan, 2006). 
Ter propósito de vida significa ter metas na vida e um senso de direção; a pessoa percebe que há sentido em sua vida presente e passada; tem crenças que dão propósito à vida; acredita que a vida é significativa e, a despeito de todos os obstáculos, tem motivação para continuar vivendo (Ryff, 1989). Janoff-Bulman e Frantz (1997, citado por Pakenham, 2005) propuseram que a adaptação positiva em face da adversidade envolve uma tentativa de encontrar sentido num evento traumático e achar algum benefício nesta experiência.

Segundo Ryff (1989), pessoas ajustadas têm alto senso de desenvolvimento e crescimento contínuo; estão abertas a novas experiências; reconhecem seu potencial de realização, e suas mudanças refletem autoconhecimento e autoeficácia. Bauer e McAdams (2004) citam Maslow (1968) para ressaltar que pessoas orientadas para o crescimento ou para a segurança em suas vidas têm melhor saúde psicológica e senso mais alto de bem-estar psicológico. Para psicólogos de orientação humanista, as pessoas que pensam na vida principalmente em termos de certos valores (por exemplo, inteireza, justiça, autonomia, empatia) estão no caminho do crescimento pessoal para maiores níveis de saúde psicológica e bem-estar.

Outro aspecto importante associado ao ajustamento psicológico é o conceito de geratividade de Erikson (1950). O autor propôs uma teoria do desenvolvimento psicossocial que descreve tarefas a serem realizadas em diferentes estágios da vida. A geratividade é um conceito amplo; segundo Erikson (1950), é um tema típico da meia-idade. É definida como deixar um legado espiritual para as próximas gerações e vista como um tema central da velhice (Erikson, Erikson \& Kvinick, 1986).

A realização da geratividade é um desafio evolutivo que contrasta com o seu oposto, a estagnação, e que deve ser enfrentado durante a vida adulta, mas de modo especial nos anos mais avançados da vida humana. A resolução deste conflito entre geratividade e estagnação resulta na força básica que é o cuidado. O esforço vital do cuidado é a principal conquista resultante da resolução do conflito, estendido para o que foi gerado pelo amor (os filhos), pela necessidade (o cuidado dos pais) ou por escolha (a atuação profissional como educador). Para ser gerativo é preciso dedicar-se a algo, acreditar e se empenhar. O sentido da geratividade é de preservação da espécie, de deixar sua marca no mundo. Para McAdams, Hart e Maruna (1998), a geratividade se expressa em termos de preocupações e de ações, ambas correspondentes às dimensões criar ou gerar, manter e oferecer.
Indivíduos bem-ajustados têm mais habilidade para adaptar-se às demandas sociais e emocionais da vida cotidiana (Bauer \& McAdams, 2004). Mesmo na presença de perdas, que podem ser reduzidas ou ampliadas de acordo com as condições da pessoa, o ser humano sempre aspirou a viver longamente, com autonomia e com boa saúde, tendo uma boa velhice.

\section{PERSPECTIVA DE VELHICE PESSOAL E VELHICE BEM-SUCEDIDA}

O significado da velhice é construído a partir da percepção pessoal, por intermédio dos valores e experiências, crenças e atitudes que cada um internaliza, e também a partir da história interacional do indivíduo com eventos contextuais de natureza reforçadora, avaliativa e diretiva (Neri, 1991).

Por ser construído socialmente, o envelhecimento adquire significados diferentes, relacionados ao gênero, à etnia, à cultura e às condições específicas de cada indivíduo. Apesar da variabilidade, o significado de envelhecer foi compartilhado entre pessoas da cultura ocidental, e este fato pode influenciar as percepções individuais de envelhecimento ao longo do tempo (Levy, 2003, citado por Harrison, 2004).

$\mathrm{O}$ envelhecimento é um processo adaptativo que depende da interação de fatores genéticos, biológicos e socioculturais. As atitudes em relação à velhice refletem os conhecimentos do senso comum e os conhecimentos científicos disponíveis sobre essa fase da vida humana. Até os anos 1960, entre leigos e profissionais, predominava um ponto de vista essencialmente negativo sobre a velhice, porém sempre existiram expectativas de controlar os efeitos deletérios da velhice, de estender a vida e prolongar a juventude. Tais expectativas ganharam destaque na ciência e nas sociedades quando o envelhecimento populacional e a possibilidade de envelhecer bem se tornaram nítidas realidades em muitos países. Desde então, vem crescendo o número de indivíduos e sociedades que investem numa velhice saudável ou que, pelo menos, reconhecem a necessidade de fazê-lo (Resende, 2001).

Houve uma evolução nas atitudes encontradas em pesquisas nos últimos 50 anos. Neri, Cachioni e Resende (2002) observam que, de maneira geral, nas pesquisas apresentadas, os adultos maduros e os idosos mostram atitudes e crenças mais positivas em relação à velhice. Indivíduos não idosos apresentaram expectativas muito positivas quanto a se manterem ativos, produtivos e valorizados na velhice, e todos os idosos e não idosos mostraram atitudes mais negativas em relação aos aspectos que definem agência humana, 
funcionalidade cognitiva e inserção social. Segundo essas autoras, os dados relatados contrariam o senso comum segundo o qual predominam atitudes negativas em relação aos idosos e ao envelhecimento na sociedade brasileira. Sugerem que há muitas possibilidades quando se trata das cognições, dos sentimentos e das experiências diretas de envelhecimento, e que as atitudes e crenças de homens e mulheres em diversas faixas de idade e em diferentes níveis de ocupação e escolaridade refletem essa variabilidade.

A preponderância de estereótipos negativos sobre o envelhecimento na sociedade não garante, automaticamente, a interiorização destes estereótipos, nem de autopercepções negativas sobre o envelhecimento. Dentro da categoria de velhos, subgrupos podem ter diferentes exposições aos estereótipos do envelhecimento. Como um exemplo, indivíduos mais velhos com surdez descreveram atitudes mais positivas em relação ao envelhecimento do que indivíduos sem incapacidade auditiva (Levy \& Langer, 1994).

Resende (2001) estudou 242 pessoas com deficiência física que tinham entre 24 e 39 anos de idade. Foram investigadas as atitudes em relação aos idosos, às pessoas com deficiência física e à própria velhice. A análise estatística mostrou superioridade de atitudes positivas e de atitudes moderadas ou pouco negativas em relação aos três objetos. Em relação aos idosos, as atitudes mais positivas foram: das mulheres, dos sujeitos de baixo nível de escolaridade e das pessoas com deficiências congênitas; e as atitudes mais negativas foram as dos sujeitos com nível mais alto de dependência. Atitudes negativas predominaram nos domínios da escala definidos como agência, cognição e relações sociais.

No tocante às pessoas com deficiência física, as atitudes mais positivas foram das mulheres, dos divorciados e dos que conviviam com a deficiência havia mais de 30 anos. Foram encontradas atitudes negativas entre dependência física e atitudes negativas em relação a pessoas com deficiência física. As atitudes em relação à própria velhice foram mais positivas do que as relativas aos idosos. Quanto mais positivas as atitudes em relação às pessoas com deficiência, mais positivas as atitudes em relação aos idosos. Segundo Resende (2001), os resultados indicam a presença de elementos autoprotetores e de processos adaptativos mediados por mecanismos de comparação social. Além disso, sugerem o controle por conhecimentos socialmente compartilhados sobre a natureza e a sequência das mudanças evolutivas (Resende \& Neri, 2005).
Esta pesquisa pretendeu contribuir para a compreensão do processo do envelhecimento psicológico de adultos com deficiência física, congênita ou adquirida. Considerando-se os resultados de pesquisa anterior (Resende, 2001), parece importante perguntar: as pessoas com deficiência física apresentam desenvolvimento pessoal positivo e uma perspectiva positiva de envelhecimento? - ou seja, será que adultos com deficiência física estão envelhecendo bem, com ajustamento psicológico?

Neste, sentido, a pesquisa teve os seguintes objetivos: a) descrever as autopercepções de pessoas com deficiência física, quanto ao próprio ajustamento psicológico, ajustamento esse indicado por medidas de autonomia, domínio, autoaceitação, crescimento pessoal, propósito, relações positivas com os outros e geratividade; b) verificar sua perspectiva de velhice pessoal; c) caracterizar relações entre o senso de ajustamento psicológico e a perspectiva de velhice pessoal.

\section{MÉTODO}

\section{Participantes ${ }^{1}$}

Esta pesquisa foi realizada com uma amostra intencional e sistemática retirada do universo da Associação dos Paraplégicos de Uberlândia (69 pessoas) e do Programa de Atendimento à Pessoa com Deficiência, da Faculdade de Educação Física da Universidade Federal de Uberlândia (21 pessoas). Os critérios de inclusão para participação na pesquisa foram: ter idade acima de 25 anos; ter deficiência física e utilizar auxílio ortopédico (cadeira de rodas, muleta, órtese ou andador) para realizar as atividades básicas e instrumentais de vida diária; ser alfabetizado; não apresentar deficiência mental ou comprometimento cognitivo; comparecer aos serviços das instituições nos meses de julho a outubro de 2005.

Participaram 90 pessoas, de ambos os sexos, com idade entre 25 e 84 anos . Os participantes eram adultos jovens $(45,5 \%)$ ou adultos de meia-idade $(44,4 \%), 56,7 \%$ dos quais do sexo masculino; $42,2 \%$ eram solteiros e $40 \%$ casados; $33,3 \%$ tinham escolaridade correspondente ao ensino básico e 31,3\% ao ensino médio; 58,9\% não trabalhavam; $62,2 \%$ eram aposentados e, entre estes, $85,2 \%$ o eram por invalidez.

1 Seguindo a Resolução 196/96 do Conselho Nacional de Saúde, sobre a pesquisa com seres humanos, este projeto foi submetido a apreciação do Comitê de Ética em Pesquisa da UFU, tendo sido aprovado, conforme Parecer nº 203/05. 
A maioria reconhecia possuir uma deficiência $(92,2 \%) ; 55,6 \%$ acreditavam que o grau de sua deficiência era moderado e o restante da amostra dividiu-se entre a avaliação de que a deficiência que tinham era grave ou leve; 93,3\% tinham deficiência física adquirida, $51,8 \%$ dos quais por doença, $39,8 \%$ por acidentes, $8,4 \%$ outros motivos. A deficiência foi adquirida na infância $(42,1 \%)$ ou na vida adulta jovem $(28,4 \%)$, entre 20 e 40 anos. Para realizar as atividades básicas da vida diária, a maioria dos participantes $(94,4 \%)$ relatou contar com a ajuda de outras pessoas, $65 \%$ das quais eram da família; $67,0 \%$ não apresentavam nenhuma doença crônica, mas as pessoas com doenças crônicas eram mais velhas do que as que não as tinham $(\mathrm{p}<0,05)$.

\section{Procedimentos}

Foram feitas entrevistas por pessoal treinado, nas instituições frequentadas pelos participantes, em dias de seu comparecimento para atividades terapêuticas ou de lazer, a partir de um protocolo que levantou os seguintes dados de autorrelato:

a) ficha de informações sociodemográficas, contendo dados de identificação;

b) ficha de informações sobre a deficiência física, contendo dados sobre a deficiência, nome e origem (congênita ou adquirida); se adquirida, idade e motivo da aquisição da deficiência; utilização de auxílio ortopédico e de outros tipos de apoio;

c) Inventário Sheppard de Atitudes em Relação à Velhice Pessoal (Neri, 1991), que avalia atitudes em relação à velhice por meio de 20 afirmações, cujas respostas devem ser dadas numa escala Likert de quatro pontos ("discordo muitíssimo" a "concordo muitíssimo") $(\alpha=0,724)$;

d) Escala de Desenvolvimento Pessoal (Queroz \& Neri, 2005), que consiste em 30 itens, cujas respostas devem ser dadas numa escala de cinco pontos ("pouquíssimo" a "muitíssimo"): 18 itens referem-se aos domínios relações positivas com outros, autonomia, propósito de vida, crescimento pessoal, autoaceitação, domínio sobre o ambiente, extraídas do modelo de Ryff (1989), e 12, ao conceito de geratividade e suas dimensões criar, manter e oferecer, estas referenciadas à teoria de Erikson (1950) e ao modelo de McAdams e St Aubin (1992) $(\alpha=0,901)$.

\section{RESULTADOS}

Para descrever o perfil da amostra segundo as variáveis em estudo, foram feitas tabelas de freqüência das variáveis categóricas e medidas de posição e dispersão. Para comparação dos escores entre dois grupos foi utilizado o teste de Mann-Whitney; para três ou mais grupos foi utilizado o teste de KruskalWallis. Para analisar a correlação entre variáveis ordinais foi utilizado o coeficiente de correlação de Spearman. Para analisar a relação entre as variáveis: idade, gênero, escolaridade, profissão, estado civil, tipo e origem da deficiência, tempo de convivência com a deficiência com ajustamento psicológico e perspectiva de velhice pessoal foi utilizada a Análise de Regressão Linear, com modelos univariado e multivariado, estes com critério Stepwise de seleção de variáveis. O nível de significância adotado para os testes estatísticos foi de 5\% ( $\mathrm{p}<0,05)$.

Com relação ao ajustamento psicológico indicado pelos escores na EDEP, os participantes apresentaram pontuação positiva $(\overline{\mathrm{X}}=3,88 \mathrm{e} \mathrm{DP}=0,52)$. Os resultados indicaram que os participantes viam-se em crescimento pessoal ( $\bar{x}=4,20$ e DP $=0,58)$ e com metas de vida $(\bar{x}=4,16$ e DP $=0,65)$. Apresentaram boa autoaceitação ( $\bar{x}=3,95$ e DP $=0,80)$, bom senso de domínio sobre o ambiente $(\bar{x}=3,94 \mathrm{e} \mathrm{DP}=0,75)$ e bom senso de autonomia $(\bar{x}=3,90$ e DP $=0,69)$. Além disso, disseram manter relações positivas com os outros $\quad(\bar{x}=3,66 \mathrm{e} \mathrm{DP}=0,74) . \quad$ Quanto à geratividade, na dimensão ofertar, a média foi de 3,92 $(\mathrm{DP}=0,65)$; em manter, a média foi de $3,59(\mathrm{DP}=0,81)$ e em criar, de 3,54 (DP=0,85).

Foi calculada a média das pontuações apresentadas pela amostra nos itens da escala que avaliavam atitudes em relação à velhice pessoal (Inventário Sheppard). Resultou a média de 2,13 $(\mathrm{DP}=0,31)$, valor que indica que as respostas foram, em geral, positivas. Na dimensão "é possível ser feliz na velhice", encontrou-se a média de 2,07 ( $\mathrm{DP}=0,31)$. $\mathrm{Na}$ dimensão "a velhice prenuncia dependência, morte e solidão", a média foi de $2,35 \quad(\mathrm{DP}=0,54)$. $\mathrm{Na}$ dimensão "é melhor morrer cedo do que sentir a angústia e solidão da velhice" obteve-se uma média de $2,10(\mathrm{DP}=0,61)$. Quanto à dimensão "a velhice pode propiciar sentimentos de integridade", obteve-se a média de $1,91(\mathrm{DP}=0,52)$.

A partir do teste de correlação de Spearman, considerando $\mathrm{p} \leq 0,05$, as comparações entre as medidas de ajustamento psicológico e de atitudes em relação à velhice pessoal revelaram que, de forma geral, quanto mais alto o escore em desenvolvimento pessoal, mais positiva a perspectiva de velhice 
pessoal, exceto para o domínio "é melhor morrer cedo do que sentir a angústia e a solidão da velhice”. Os indivíduos com escores mais altos nos domínios propósito na vida $(\mathrm{r}=-0,31226)$, senso de crescimento pessoal $(r=-0,28402)$, relações positivas com os outros $(\mathrm{r}=-0,23534)$ e geratividade nos domínios criar $(\mathrm{r}=-$ 0,26290) e oferecer ( $\mathrm{r}=-0,28093)$ foram os que pontuaram mais alto no domínio "é possível ser feliz na velhice”. A partir do teste de correlação de Spearman, considerando $\mathrm{p} \leq 0,05$, as pessoas que menos acreditam que a velhice prenuncia dependência, morte e solidão foram as que apresentaram pontuação mais alta em domínio sobre o ambiente ( $\mathrm{r}=-0,26788)$, propósito na vida ( $\mathrm{r}=-$ $0,41236)$, crescimento pessoal $(\mathrm{r}=-0,28016)$, relações positivas com os outros ( $\mathrm{r}=-0,29750)$, autoaceitação $(\mathrm{r}=-0,41640)$ e geratividade nos domínios criar $(\mathrm{r}=-$ $0,36514)$ e oferecer $(r=-0,34408)$. A crença de que a velhice pode propiciar sentimentos de integridade foi tanto maior quanto maiores o senso de propósito na vida ( $r=-0,33731)$ e a autoaceitação $(r=-0,25672)$.

Considerando p-valor referente ao teste de Mann-Whitney, em comparação com os homens, as mulheres apresentaram menos senso positivo de desenvolvimento pessoal $(\mathrm{p}=0,050)$. As mulheres também apresentaram mais baixo senso de crescimento pessoal e menos relações positivas com outros. O teste de Mann-Whitney indicou que os indivíduos com menos de 40 anos apresentam maior senso de autonomia $(p=0,046)$ do que os grupos mais velhos. A partir da correlação de Spearman, considerando $\mathrm{p} \leq 0,05$, quanto mais jovens, maior o senso de autonomia ( $\mathrm{r}=-0,24058)$, crescimento pessoal $(\mathrm{r}=-0,27016)$ e geratividade no domínio criar ( $\mathrm{r}=-0,22578)$. Quanto maior o tempo de convivência com a deficiência, mais as pessoas tenderam a opinar que "É melhor morrer cedo do que sentir a angústia e a solidão da velhice" $(\mathrm{r}=0,25243)$.

Foram feitas análises de regressão univariada (ver Tabela 1) e múltipla (ver Tabela 2), que revelaram as seguintes associações significativas: quanto maior a idade, mais baixo o senso de ajustamento pessoal; relação conjunta significativa entre ajustamento psicológico, do tipo de deficiência (congênita ou adquirida), e perspectiva de velhice pessoal;ou seja, os indivíduos com atitudes mais positivas em relação ao envelhecimento pessoal foram aqueles com deficiência congênita e com escores mais altos na medida de ajustamento psicológico.

Tabela 1. Resultados da Análise de Regressão Linear Univariada para Escore Total da EDEP

\begin{tabular}{|c|c|c|c|c|}
\hline Variável & Categorias & p-valor & $\mathrm{R}^{2}(\%)$ & Estimativa (E.P.)* \\
\hline $\begin{array}{l}\text { Idade } \\
\text { (faixas) }\end{array}$ & $\begin{array}{l}<40 \text { anos } \\
>=40 \text { anos }\end{array}$ & $\begin{array}{c}-- \\
0.440\end{array}$ & 0.68 & $\begin{array}{c}3.93(0.08) \\
-0.09(0.11)\end{array}$ \\
\hline Idade & variável contínua & 0.045 & 4.51 & $\begin{array}{c}4.26(0.20) \\
-0.01(0.004)\end{array}$ \\
\hline Sexo & $\begin{array}{l}\text { Masculino } \\
\text { Feminino }\end{array}$ & $\begin{array}{c}--- \\
0.096\end{array}$ & 3.12 & $\begin{array}{c}3.96(0.07) \\
-0.19(0.11)\end{array}$ \\
\hline Escolaridade & $\begin{array}{l}\text { Básico+Fundamental } \\
\text { Médio+Superior }\end{array}$ & 0.444 & 0.67 & $\begin{array}{l}3.84(0.07) \\
0.09(0.11)\end{array}$ \\
\hline Estado Civil & $\begin{array}{l}\text { Sem Companheiro } \\
\text { Com Companheiro }\end{array}$ & $\begin{array}{c}--- \\
0.885\end{array}$ & 0.02 & $\begin{array}{r}3.89(0.07) \\
-0.02(0.11)\end{array}$ \\
\hline Tipo de Deficiência & $\begin{array}{l}\text { Adquirida } \\
\text { Congênita } \\
\text { Por Doenca }\end{array}$ & $\begin{array}{c}--- \\
0.127 \\
---\end{array}$ & 2.63 & $\begin{array}{l}3.86(0.06) \\
0.34(0.22) \\
3.87(0.08)\end{array}$ \\
\hline Origem da Deficiência & $\begin{array}{c}\text { Por Acidente } \\
\text { Outros+Congênita }\end{array}$ & $\begin{array}{l}0.868 \\
0.865\end{array}$ & 0.05 & $\begin{array}{l}0.02(0.12) \\
0.03(0.16)\end{array}$ \\
\hline
\end{tabular}

$* \mathrm{R}^{2}=$ coeficiente de determinação (\% de variabilidade da variável resposta explicada pela variável independente); E.P.=Erro Padrão; Estimativa = valores do intercepto $\left(1^{\circ}\right.$ valor $)$ e do coeficiente angular $\left(2^{\circ}\right.$ valor $)$ na reta de regressão $\mathrm{Y}=\mathrm{a}+\mathrm{bX}$.

Variável Dependente: Escore Total da EDEP (ajustamento psicológico); n=90 participantes.

Tabela 2. Resultados da Análise de Regressão Linear Múltipla para Escore da EDEP (n=90).

\begin{tabular}{ccccc}
\hline Variáveis Selecionadas & Categorias/Níveis & p-valor & $\mathbf{R}^{\mathbf{2}}(\boldsymbol{\%})$ & Estimativa (E.P.)* \\
\hline Idade & variável contínua & $\mathbf{0 . 0 4 5}$ & 4.51 & $4.26(0.20)$ \\
& & & & $-0.01(0.004)$ \\
\hline
\end{tabular}

$* \mathrm{R}^{2}=$ coeficiente de determinação parcial; E.P.=Erro Padrão; Estimativa = valores do intercepto $\left(1^{\circ}\right.$ valor $)$ e do coeficiente angular $\left(2^{\circ}\right.$ valor $)$ na reta de regressão. Variável Dependente: Escore Total da EDEP. Critério Stepwise de seleção de variáveis. $\mathrm{R}^{2}$ Total=4.51\% 
Tabela 3. Resultados da Análise de Regressão Linear Univariada para Escore de Sheppard

\begin{tabular}{|c|c|c|c|c|}
\hline Variável & Categorias & p-valor & $\mathrm{R}^{2}(\%)$ & Estimativa (E.P.)* \\
\hline $\begin{array}{l}\text { Idade } \\
\text { (faixas) }\end{array}$ & $\begin{array}{l}<40 \text { anos } \\
>=40 \text { anos }\end{array}$ & $0 .-\overline{-}$ & 0.42 & $\begin{array}{l}2.10(0.05) \\
0.04(0.07)\end{array}$ \\
\hline Idade & variável contínua & 0.212 & 1.76 & $\begin{array}{c}1.98(0.12) \\
0.01(0.003)\end{array}$ \\
\hline Sexo & $\begin{array}{l}\text { Masculino } \\
\text { Feminino }\end{array}$ & 0.468 & 0.60 & $\begin{array}{l}2.11(0.04) \\
0.05(0.07)\end{array}$ \\
\hline Escolaridade & $\begin{array}{l}\text { Básico+Fundamental } \\
\text { Médio+Superior }\end{array}$ & 0.060 & 3.96 & $\begin{array}{l}2.18(0.04) \\
-0.12(0.06)\end{array}$ \\
\hline Estado Civil & $\begin{array}{l}\text { Sem Companheiro } \\
\text { Com Companheiro }\end{array}$ & $\begin{array}{c}--- \\
0.885\end{array}$ & 0.02 & $\begin{array}{l}2.13(0.04) \\
-0.01(0.07)\end{array}$ \\
\hline Tipo de Deficiência & $\begin{array}{l}\text { Adquirida } \\
\text { Congênita } \\
\text { Por Doenca }\end{array}$ & $\begin{array}{c}--- \\
0.012 \\
---\end{array}$ & 6.91 & $\begin{array}{l}2.15(0.03) \\
-0.32(0.13) \\
2.20(0.05)\end{array}$ \\
\hline Origem da Deficiência & $\begin{array}{c}\text { Por Acidente } \\
\text { Outros+Congênita }\end{array}$ & $\begin{array}{l}0.140 \\
\mathbf{0 . 0 3 9}\end{array}$ & 5.55 & $\begin{array}{l}-0.10(0.07) \\
-0.20(0.09)\end{array}$ \\
\hline Ajustamento Psicológico (escore total) & variável contínua & $<0.001$ & 18.31 & $\begin{array}{l}3.11(0.22) \\
-0.25(0.06)\end{array}$ \\
\hline
\end{tabular}

$* \mathrm{R}^{2}=$ coeficiente de determinação (\% de variabilidade da variável resposta explicada pela variável independente); E.P.=Erro Padrão; Estimativa = valores do intercepto (1o valor) e do coeficiente angular (2o valor) na reta de regressão $\mathrm{Y}=\mathrm{a}+\mathrm{bX}$.

Variável Dependente: Escore Total de Sheppard (perspectiva de velhice pessoal); n=90 participantes.

Tabela 4. Resultados da Análise de Regressão Linear Múltipla para Escore de Sheppard

\begin{tabular}{ccccc}
\hline Variáveis Selecionadas & Categorias/Níveis & p-valor & $\mathbf{R}^{\mathbf{2}}(\boldsymbol{\%})$ & Estimativa (E.P.)* \\
\hline \multirow{2}{*}{ Ajustamento Psicológico (escore total) } & variável contínua & $\mathbf{< 0 . 0 0 1}$ & 18.31 & $3.05(0.22)$ \\
Tipo de Deficiência & Adquirida & $---23(0.06)$ & $-0.24(0.12)$ \\
\hline
\end{tabular}

$* \mathrm{R}^{2}=$ coeficiente de determinação parcial; E.P.=Erro Padrão; Estimativa $=$ valores do intercepto $\left(1^{\circ}\right.$ valor $)$ e do coeficiente angular $\left(2^{\circ}\right.$ valor $)$ na reta de regressão. Variável Dependente: Escore Total de Sheppard. Critério Stepwise de seleção de variáveis. $\mathrm{R}^{2}$ Total=22.16\%

\section{DISCUSSÃO}

Esta pesquisa demonstrou indícios de ajustamento pessoal em indivíduos adultos e idosos, indicando que os participantes estão adaptados à deficiência. Apresentaram boa autoaceitação, bom senso de domínio sobre o ambiente e de autonomia e bom senso de que mantêm relações positivas com os outros. Quanto à geratividade, os participantes relataram preocupações pelo bem-estar atual e futuro da próxima geração nas dimensões conceituais ofertar, manter e criar.

$\mathrm{O}$ ajustamento pessoal a uma deficiência com início na vida adulta é um longo e multifacetado processo que envolve ajustamento psicossocial e físico. Para Dijkers (2005), indivíduos que se adaptam a deficiências físicas por exemplo, à decorrente de lesão medular - possuem e relatam melhor qualidade de vida, especialmente os que moram em países desenvolvidos - que têm acesso a cuidados clínicos, equipamentos adaptados, apoio econômico e social - e os que vivem em cidades onde casas, edifícios, espaços públicos e transporte são acessíveis. Oliveira (2004) descreve que a expectativa de vida de indivíduos com deficiência reflete a sua adaptação e o seu ajustamento. A adaptação é uma atividade integrada à sobrevivência e deve ser considerada nos níveis fisiológico, psicológico e social do funcionamento individual. Por outro lado, o ajustamento à incapacidade é uma tentativa da pessoa de encontrar o equilíbrio ante as pressões externas e internas.

De maneira geral, os resultados aqui encontrados apontam uma tendência dos participantes a acreditarem numa perspectiva de velhice pessoal positiva. Para Seeman (2000), uma visão mais positiva do envelhecimento pode ajudar os indivíduos a alcançar e manter níveis mais altos de funcionamento à medida que envelhecem. Em pesquisa com 660 pessoas com 60 anos ou mais, Levy, Slade, Kunkel e Kasl (2002) encontraram que indivíduos mais velhos, com autopercepções positivas sobre o envelhecimento, vivem mais que aqueles com visão mais negativa sobre a velhice; ou seja, uma atitude positiva em relação à velhice e o desejo de uma vida longa contribuem para que a pessoa viva mais e melhor.

Os resultados da presente pesquisa indicaram que, quanto maior o senso de desenvolvimento pessoal, mais positiva é a perspectiva da própria velhice. Os indivíduos que tinham mais propósito na vida, senso de crescimento, relações positivas com os outros e geratividade (nos domínios criar e oferecer) são aqueles que mais acreditam que é possível ser feliz na velhice. A perspectiva de 
velhice pessoal em pessoas com deficiência está relacionada à maneira como encaram a própria deficiência. Em pesquisa com 153 pessoas com deficiência, Albrecht e Devlieger (1999) encontraram que $54,3 \%$ dos participantes responderam ter excelente ou ótima qualidade de vida, portanto, que tinham uma atitude positiva em relação à deficiência física. Segundo estes autores, entre os fatores que contribuíram para a percepção positiva da deficiência estão os seguintes: continuarem tendo controle sobre o corpo, a mente e a vida, apesar de conviverem com algumas limitações físicas e/ou mentais; a possibilidade de continuar realizando tarefas; sentimentos de maturidade que adquiriram e a satisfação em dar suporte emocional a outras pessoas; a importância da fé e a redescoberta da espiritualidade, que lhes davam força, direção e sentido à vida; a utilização da experiência de ser deficiente para esclarecer e reorientar suas vidas; a força interna e a resiliência, que, para essas pessoas, vieram por meio da revisão de valores e de uma visão equilibrada entre mente, corpo e espírito, no ajustamento no modo de ser, que proporcionou um bom senso de perspectiva de vida. Ademais, a deficiência proporcionou uma fonte de valores e metas, ímpeto para crescimento psicológico e oportunidade para mudar e amadurecer.

De acordo com Gallagher e MacLachlan (2004), apesar de o aspecto mais presente na literatura ser o ajustamento aos aspectos físicos, é necessário contemplar a possibilidade de que as pessoas reparem o desequilíbrio e assim promovam o próprio ajustamento, por meio de variáveis psicossociais. De acordo com Oaksford, Frude e Cuddihy (2005), há evidência crescente de que eventos estressantes nem sempre terminam em resultados negativos e de que algumas pessoas se tornam mais fortes ou crescem psicologicamente porque passaram por situações em que precisaram enfrentar eventos estressantes. Estes autores afirmam que, no caso de pessoas com deficiência física, as pesquisas sugerem que os indivíduos podem fazer mais do que sobreviver à sua deficiência: podem tornar-se resilientes e adaptar-se à sua condição, se lutarem pelo alcance de metas pessoais e pelo ajustamento psicológico positivo.

Nesta pesquisa, as mulheres apresentaram senso menos positivo de desenvolvimento pessoal do que os homens, ao contrário dos dados encontrados em pesquisas australianas. Por exemplo, Dorsett (2001) afirma que as mulheres parecem aceitar melhor a incapacidade do que os homens, embora esse dado precise de refinamento, visto que, de modo geral, o número de mulheres que participam de estudos sobre deficiência física e incapacidade é menor do que o de homens, possivelmente pela mesma razão apontada acima.
Neri (2006) afirma que a relação entre os padrões de independência e dependência, em associação com gênero, comporta variações e sobreposições que ocorrem em função de eventos culturais e de personalidade. Segundo a autora, os especialistas apontam para uma dialética contínua entre a dependência e a autonomia e para o reconhecimento de que, mesmo na idade adulta e na velhice, as pessoas não se libertam das relações e da necessidade de assistência dos outros, e sim, empenhamse na construção de um self envolvido e fazem adaptações para manejar as dificuldades.

Os indivíduos da amostra estudada com menos de 40 anos disseram ter senso de autonomia mais do que o fizeram os grupos mais velhos. Comparações sociais ajudam na percepção de si mesmo a partir do outro. Os indivíduos avaliam-se, em parte, baseados na comparação com os outros. As comparações "para baixo", ou seja, com indivíduos que estão em pior condição, têm função adaptativa, porque protegem o self, melhoram e salvam a autoestima em situações de inferioridade (Kwan, Love, Ryff \& Essex, 2003).

Para Hughes, Robinson-Whelen, Taylor, Swedlund e Nosek (2004), a autoestima tem um importante papel no bem-estar de mulheres com limitações físicas. Infelizmente, a autoestima em mulheres com deficiência física e condições crônicas de saúde pode ficar comprometida por diversas razões, tais como a internalização de estereótipos e estigmas sociais, exclusão e a desvalorização que a sociedade impõe às pessoas estigmatizadas (Goffman, 1988).

As pessoas com atitudes mais positivas em relação ao envelhecimento pessoal foram as que tinham deficiência congênita e as que apresentavam melhores escores em ajustamento psicológico. Pavarini e Neri (2000) relatam que as pessoas com deficiência física podem ativar mecanismos de adaptação para enfrentar perdas em funcionalidade, por intermédio de recursos tecnológicos e de apoios psicológicos e sociais. Como as pessoas com deficiência congênita deste estudo já convivem com a deficiência há pelo menos 25 anos (idade mínima para participar dessa pesquisa), é esperado que estejam adaptadas à sua condição (Resende, 2004; Resende, 2005).

Segundo Dorsett (2001), o ajustamento psicológico é um processo dinâmico. Embora haja pouco consenso na literatura sobre os elementos mais importantes para alcançá-lo após uma deficiência física, os mais encontrados são segurança financeira, nível educacional ou socioeconômico mais alto, acessibilidade ambienta, idade menos avançada quando da aquisição da deficiência, a condição de casado e rede de relações apoiadora. 
a) Com mais recursos disponíveis, aumenta a possibilidade de adequar o ambiente (empregados, adequação de residência, utilização de equipamentos tecnológicos para ajudar na independência e aumento na qualidade de vida). Neste sentido é possível que a pessoa tenha uma percepção mais forte de controle sobre a própria vida.

b) Pessoas com nível educacional ou socioeconômico mais alto apresentam melhor ajustamento e referem maior satisfação com a vida, ao contrário das pessoas com escolaridade mais baixa, que frequentemente relatam maior número de internações.

c) Quanto à acessibilidade ambiental, notou-se que mobilidade pessoal e mais acesso aos meios de transporte estão positivamente correlacionados a autoconceito mais desenvolvido e a melhor autoestima, mais envolvimento em atividades sociais e elevados níveis de bem-estar subjetivo, fatores que, em conjunto, indicam melhor ajustamento psicológico.

d) Envelhecer com uma deficiência parece favorecer o ajustamento. Pessoas mais jovens parecem aceitar melhor a incapacidade, talvez por serem mais flexíveis em suas atitudes em relação à vida e por serem mais abertas a novos e diferentes modos de realizar tarefas.

e) Ser casado geralmente está relacionado a melhor qualidade de vida e a arranjos de vida mais independentes. Além disso, viver numa relação conjugal estável geralmente implica maior disponibilidade de ajuda instrumental e apoio emocional.

f) O apoio social é uma variável importante, facilitadora de resultados positivos para o bem-estar subjetivo de pessoas com deficiência física. Indivíduos que têm altos níveis de apoio social e estão satisfeitos com a qualidade de sua rede de relações têm maior senso de bem-estar (Caprara \& Steca, 2005).

\section{CONSIDERAÇÕES FINAIS}

Essa pesquisa contribuiu para mostrar que a pessoa com melhor ajustamento pessoal parece ter também maior perspectiva positiva de envelhecimento. Ajustar-se a uma deficiência é um processo social complexo e requer ajuste pessoal e social contínuo. Como o foco de cuidado em saúde tem se voltado para a inclusão da pessoa com deficiência na sociedade, torna-se fundamental para os profissionais de saúde que trabalham com reabilitação compreenderem como as pessoas se ajustam e vivem o dia-a-dia com uma deficiência.
É necessário os profissionais que trabalham com pessoas com deficiência ampliem suas visões sobre a relação entre dependência e independência, respeitando a autonomia de seu cliente e entendendo que o trabalho bem-sucedido no auxílio ao ajustamento implica no reconhecimento do significando de seu impacto na vida do indivíduo. Os profissionais de saúde devem ampliar seu conhecimento e estender seu ponto de vista para o entendimento da deficiência como mais uma característica da pessoa.

Por isso, conhecer as habilidades para adaptar-se às mudanças circunstanciais indicadoras de ajustamento e, consequentemente, de um envelhecimento positivo, é fundamental para um bom planejamento de intervenções na área da educação e da saúde, além de auxiliar no planejamento de políticas públicas que poderão reduzir custos no campo da reabilitação e, consequentemente, na melhora da saúde pública.

\section{REFERÊNCIAS}

Albrecht, G. L., \& Devlieger, P. J. (1999). The disability paradox: high quality of life against all odds. Social Science and Medicine, 48(8), 977-988.

Bauer, J. J., \& McAdams, D. P. (2004). Growth goals, maturity, and well-being. Developmental Psychology, 40(1), 114127.

Caprara, G. V., \& Steca, P. (2005). Affective and social selfregulatory efficacy beliefs as determinants of positive thinking and happiness. European Psychologist, 10(4), 275-286.

Cristopher, J. C. (1999). Situating psychological well-being: exploring the cultural roots of its theory and research. Journal of Counseling and Development, 77, 141-152.

Dijkers, M. P. J. M. (2005). Quality of life of individuals with spinal cord injury: A review of conceptualization, measurement, and research findings. Journal of Rehabilitation Research \& Development, 42(3), 87-110.

Dorsett, P. A. (2001). Spinal cord injury: how do people cope? Tese de Doutorado Não-Publicada, University of Queensland, Australia.

Erikson, E. H. (1950). Childhood and society. New York: W. W. Norton.

Erikson, E. H., Erikson, J. M., \& Kivnick, H. (1986). Vital involvement in old age. New York: W.W. Norton \& Company.

Folkman, S. (1984). Personal control and stress and coping processes: A theoretical analysis. Journal of Personality and Social Psychology, 46, 839-852.

Gallagher, P., \& MacLachlan, M. (2004). The Trinity Amputation and Prosthesis Experience Scales (TAPES) and quality of life in people with a lower limb amputation. Archives of Physical Medicine and Rehabilitation, 85(5), 730-736. 
Goffman, E. (1988). Estigma: notas sobre a manipulação da identidade deteriorada. Rio de Janeiro: Guanabara-Koogan.

Harrison, T. C. (2004). The meaning of aging for women with childhood onset disabilities. Dissertação de Mestrado NãoPublicada, The University of Texas at Austin.

Hughes, R. B., Robinson-Whelen, S., Taylor, H. B., Swedlund, N., \& Nosek, M. A. (2004). Enhancing self-esteem in women with physical disabilities. Rehabilitation Psychology, 49(4), 295-302.

Kwan, C. M. L., Love, G. D., Ryff, C. D., \& Essex M. J. (2003). The role of self-enhancing evaluations in a successful life transition. Psychology and Aging, 18(1), 312.

Levy, B. \& Langer, E. (1994). Aging free from negative stereotypes: successful memory in China and among the American deaf. Journal of Personality and Social Psychology, 66, 989-997.

Levy, B. R., Slade, M. D., Kunkel, S. R., \& Kasl, S. V. (2002). Longevity Increased by Positive Self-Perceptions of Aging. Journal of Personality and Social Psychology, 83(2), 261270.

McAdams, D. P., Hart, H. M., \& Maruna, S. (1998). The anatomy generativity. In: D. P. McAdams, H. M. Hart, S. Maruna, S. (Orgs.). Generativity and adult development: how and why we care for the next generation (pp. 7-43) Washington, DC: Americam Psychological Association.

McAdams, D. P., \& St. Aubin, E. (1992). A theory of generativity and its assessment through self-report, behavioral acts, and narrative themes in autobiography. Journal of Personality and Social Psychology, 62, 10031015.

Neri, A. L. (2006). Teorias psicológicas do envelhecimento: percurso histórico e teorias atuais. In: E. V. Freitas, L. Py, F. A. X. Cançado, J. Doll, \& M. L. Gorzoni (orgs.). Tratado de Geriatria e Gerontologia. $2^{\mathrm{a}}$ ed. rev. amp (pp. 58-77). Rio de Janeiro: Guanabara Koogan.

Neri, A. L. (1991). Envelhecer num país de jovens: significados de velho e velhice segundo brasileiros não idosos. Campinas, SP: UNICAMP.

Neri, A. L., Cachioni, M., \& Resende, M. C. (2002). Atitudes em relação à velhice. In: E. V. Freitas, L. Py, A. L. Neri, F. A. X. Cançado, M. L. Gorzoni, \& S. M. Rocha (orgs.) Tratado de Geriatria e Gerontologia (pp. 972-980). Rio de Janeiro: Guanabara-Koogan.

Nogueira, E. J. (2001). Rede de relações sociais: um estudo transversal com homens e mulheres pertencentes a três grupos etários. Tese de Doutorado Não-Publicada, Faculdade de Educação, UNICAMP, Campinas.

Oaksford, K., Frude, N., \& Cuddihy, R. (2005). Positive coping and stress-related psychological growth following lower limb amputation. Rehabilitation Psychology, 50(3), 266277.
Oliveira, T. R. B. (2004). Ajustamento psicossocial em pacientes com lesões traumáticas do sistema nervoso central. Tese de Doutorado Não-Publicada, Faculdade de Ciências Médicas, UNICAMP, Campinas.

Pakenham, K. I. (2005). Benefit finding in multiple sclerosis and associations with positive and negative outcomes. Health Psychology, 24(2), 123-132.

Pavarini, S. C. I., \& Neri, A. L. (2000). Compreendendo dependência, independência e autonomia no contexto domiciliar: conceitos, atitudes e comportamentos. Em Y. A. O. Duarte, M. J. D. Diogo (orgs.). Atendimento domiciliar: um enfoque gerontológico. (pp. 49-69). São Paulo: Atheneu.

Queroz, N. C., \& Neri, A. L. (2005). Bem-estar psicológico e inteligência emocional entre homens e mulheres na meia idade e velhice. Psicologia: Reflexão e Crítica,18(2), 292299.

Resende, A. P. C. (2004). Todos na cidade: o direito a acessibilidade das pessoas com deficiência física em Uberlândia. Uberlândia: EDUFU.

Resende, M. C. (2005). Deficiência e incapacidade física. A. L. Neri (org.) Palavras-chave em Gerontologia. (pp. 50-54) 2. ed. Campinas, SP: Alínea.

Resende, M. C. (2001). Atitudes em relação ao idoso, à velhice pessoal e ao portador de deficiência física em adultos portadores de deficiência física. Dissertação de Mestrado Não-Publicada, Faculdade de Educação, UNICAMP, Campinas.

Resende, M. C., \& Neri, A. L. (2005). Atitudes de adultos com deficiência física frente ao idoso, à velhice pessoal e a pessoas com deficiência física. Estudos de Psicologia, Campinas, 22(2), 123-131.

Ryff, C. D. (1989). Happiness is everything, or is it? Explorations on the meaning of psychological well-being. Journal of Personality and Social Psychology, 57(6), 10691081.

Schmitt, M. M., \& Elliott, T. R. (2004). Verbal learning ability and adjustment to recent-onset spinal cord injury. Rehabilitation Psychology, 49(4), 288-294.

Seeman, T. E. (2000). Health promoting effects of friends and family on health outcomes in older adults. American Journal of Health Promotion, 14, 362-370.

Sirois, F. M., Davis, C. G., \& Morgan, M. (2006). Learning to live with what you can't rise above: Control beliefs, symptom control, and adjustment to tinnitus. Health Psychology, 25(1), 119-123.

Recebido em 30/08/2007 Aceito em 05/08/2009

Endereço para correspondência : $\quad$ Marineia Crosara de Resende. Universidade Federal de Uberlândia, Instituto de Psicologia. Av. Pará, 1720, Campus Umuarama, CEP 38405-382, Uberlândia-MG, Brasil.

E-mail: marineiaresende@gmail.com 